\title{
A prospective study of associated surgical hazards in trans-urethral resection of bladder tumor (turbt) comparing blind versus nerve stimulator guided obturator nerve block with spinal block
}

\author{
Archana Agrawal ${ }^{1, *}$, Somnath Longani ${ }^{2}$, Jaishree Bogra ${ }^{3}$ \\ ${ }^{1}$ Associate Professor, ${ }^{2}$ Assistant Professor, ${ }^{3}$ Professor \& HOD, Dept. of Anaesthesia, Hind Institute of Medical Sciences, \\ Barabanki, Lucknow, Uttar Pradesh, India \\ *Corresponding author: \\ Email: neelabhkgmu@gmail.com
}

Received: $07^{\text {th }}$ March, 2017

Accepted: $17^{\text {th }}$ May, 2017

\begin{abstract}
Introduction: Bladder cancer is the ninth most commonly diagnosed cancer worldwide. The overall incidence has increased by $10 \%$ since last 30 years and 5 years survival rate by $50 \%$ in European countries. ${ }^{1}$ Transurethral resection of bladder tumor (TURBT) is the standard treatment given to the patients of superficial bladder tumors. During TURBT, local anatomical anesthesia may be given in order to block the obturator nerve thus avoiding the obturator jerk reflex. This study analyzes the surgical hazards in TURBT comparing blind versus nerve stimulator guided obturator nerve block with spinal block.

Materials and Methods: A prospective randomized study was done in which 30 patients at HIMS and associated hospitals, were divided into three groups to compare the surgical hazards in TURBT comparing blind versus nerve stimulator guided obturator nerve block with spinal block.

Results: 30 patients were included in this study. Mean operative time in Group 1(Obturator nerve block (ONB) during TURBT via blind technique) was 38 minutes, in Group 2 (ONB during TURBT via nerve stimulation technique) was 35 minutes and in group 3 (TURBT under spinal anesthesia only was 64 minutes. Effectiveness of blind technique of obturator nerve block (Group 1) in our study was $80 \%$ while that via nerve stimulator guided technique (Group 2) was $90 \%$.

Conclusion: This study indicates that use Obturator nerve block during TURBT via nerve stimulation technique is a better technique as compared to ONB during TURBT via blind technique. The use of nerve stimulator before ONB has better outcome.
\end{abstract}

Keywords: Bladder spasm, Carcinoma, Obturator block, Spinal block, TURBT.

\section{Introduction}

The bladder is lined by transitional epithelium which can give rise to a variety of benign and malignant neoplasms. There are three main types of neoplasm that commonly occur in bladder which include: ${ }^{2}$

1. Transitional cell (urothelial) carcinoma

2. Squamous cell carcinoma

3. Adenocarcinoma

The most common amongst the three types is the transition cell carcinoma. ${ }^{3}$ The Gold standard for diagnosing bladder cancer is biopsy obtained during Cystoscopy. ${ }^{3}$ Depth of invasion of bladder tumors guides the treatment modality to be used. Superficial tumors which do not invade the muscular layer can be dealt with modalities like resecting the localized pathology using a resectoscope. This method is called TURBT which also helps in the pathological staging of tumor. This modality is not of much use in cases of muscle invading tumors. ${ }^{4}$

\section{Clinical importance of obturator nerve}

The anatomical position of obturator nerve is important in surgeries like TURBT. It generally lies on the lateral wall of distended bladder. During TURBT, electric current from the resectoscope can get transmitted to the obturator nerve and cause adductor muscle reflex jerk. This can lead to movement of bladder towards the cautery blade and can lead to perforation of bladder. ${ }^{2-4}$
TURBT is routinely done in spinal anaesthesia but since it cannot prevent obturator jerk reflex, Narins (1957) and Hobika (1961) demonstrated the use of succinylcholine and d-tubocurarine to diminish this obturator reflex. However this method required general anaesthesia with the use of endotracheal intubation which is not accepted worldwide. ${ }^{5,6}$ Prentiss demonstrated use of regional anesthesia to block the obturator reflex during TURBT. ${ }^{7}$

\section{Materials and Methods}

This prospective study was conducted in the Department of General Surgery, Hind Medical College and associated hospitals, Barabanki, from $1^{\text {st }}$ January, 2015 to $31^{\text {st }}$ August 2016. A total of 30 patients of urinary bladder tumor with required eligibility criteria were included in this study.

\section{Eligibility Criteria}

All patients with proven lateral bladder wall tumors on USG abdomen and other investigations such as cystoscopy were included in the study. These patients underwent clinical, general, systemic examinations and the required investigational procedures. Only those patients who were fit for surgery and had no obvious neurological impairment, coagulopathy or hematological disorders were included in the study. Patients with 
history of bleeding disorder, coagulation defects, infection or psychiatric disease were not included in the study.

Depending on the type of anesthesia, the patients were divided into 3 groups as follows:

1. Group 1: Patients of lateral bladder wall tumors that underwent Obturator nerve block (ONB) during TURBT via blind technique using anatomical landmarks along with spinal anesthesia. Number of patients in this group were 10 .

2. Group 2: Patients of lateral bladder wall tumors that underwent ONB during TURBT via nerve stimulation technique using a nerve stimulator along with spinal anesthesia. Number of patients in this group were 10 .

3. Group 3: Patients of lateral bladder wall tumors that underwent TURBT under spinal anesthesia only. Number of patients in this group were 10 .

\section{Techniques}

There are two techniques of giving obturator nerve block:

\section{Blind anatomic approach}

1. After giving spinal anaesthesia, lithotomy position was made.

2. $5 \mathrm{ml}$ of local anaesthetic is given at a point $1.0 \mathrm{~cm}$ lateral and inferior to the pubic tubercle.
3. Some amount of local anesthetic is also given both $1 \mathrm{~cm}$ medially and laterally to this point.

4. If superior ramus is encountered in the path of needle, it is withdrawn and then re inserted about 30 degrees laterally and 20 degrees superiorly for 2-4 $\mathrm{cm}$.

\section{Nerve Stimulation Approach}

1. Initial steps are the same as described previously.

2. The needle after insertion is stimulated with about $1.5 \mathrm{~mA}$ electric current and the strength of adductor contraction is monitored.

3. As soon as the strong contraction of adductor muscles is observed, $1 \%$ lignocaine is injected at the exact point and kept there until the contraction ceases.

\section{Results}

In the study group of 30 patients, the male to female ratio was $2: 1$. The average age of the patients was 60 years ranging from 40 to 75 years (Graph 1). Mean age of the patients in group 1 is 66.6 years, in group 2 is 59.7 years and in group 3 is 64.8 years. Eleven patients (36\%) had single mass, twelve patients $(40 \%)$ had two masses while seven patients $(23 \%)$ had multiple masses (Table 1).

\section{Graph 1: Age Distribution}

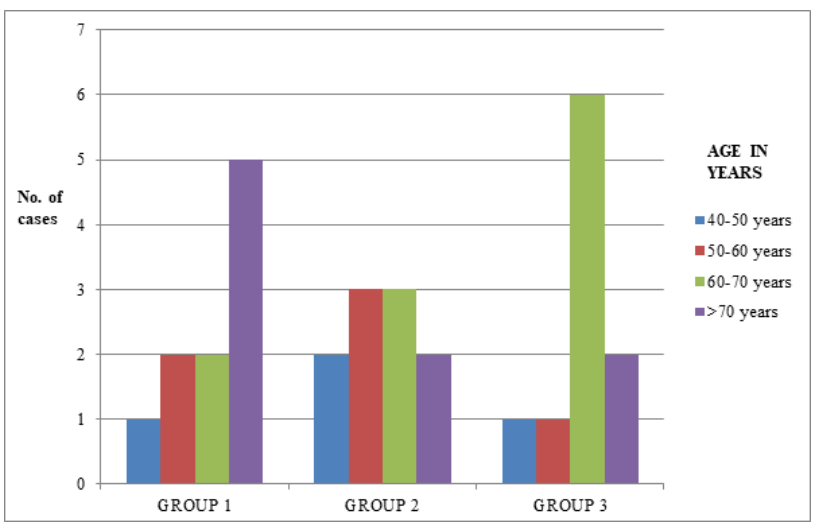

Table 1: Number of bladder masses in each group

\begin{tabular}{|l|c|c|c|c|}
\hline No. of masses & Group 1 & Group 2 & Group 3 & Total \\
\hline One & 3 & 2 & 6 & 11 \\
\hline Two & 5 & 4 & 3 & 12 \\
\hline Multiple & 2 & 4 & 1 & 7 \\
\hline Total & 10 & 10 & 10 & 30 \\
\hline
\end{tabular}

Mean operative time in group 1 was 38 minutes, in group 2 was 35 minutes and in group 3 was 64 minutes (Graph 2). Four patients in group 3 required transfusion of 1 unit of blood while only one patient in both group 1 and group 2 each required blood transfusion. Bladder spasm due to obturator nerve reflex was observed in all the patients of group 3 while only $20 \%$ patients in group 1 and $10 \%$ patients in group 2 encountered bladder spasm signifying the effectiveness of obturator nerve block (Table 2).

Effectiveness of blind technique of obturator nerve block (Group 1) in our study was $80 \%$ while that via nerve stimulator guided technique (Group 2) was 90\% (Table 3). 
Graph 2: Comparison between the groups according to the duration of surgery

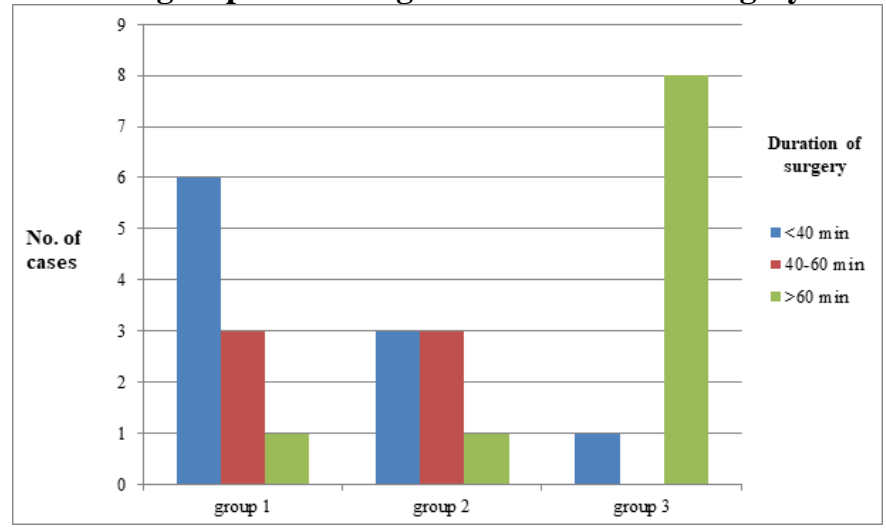

Table 2: Number of bladder spasms in all groups

\begin{tabular}{|l|c|c|c|c|}
\hline & Group 1 & Group 2 & Group 3 & Total \\
\hline None & 8 & 9 & 0 & 17 \\
\hline$<10$ & 2 & 1 & 1 & 4 \\
\hline$>10$ & 0 & 0 & 9 & 9 \\
\hline Total & 10 & 10 & 10 & 30 \\
\hline
\end{tabular}

Table 3: Effectiveness of obturator nerve block in group 1 and group 2

\begin{tabular}{|l|c|c|c|}
\hline & Group 1 & Group 2 & Total \\
\hline Effective & 8 & 9 & 17 \\
\hline Not effective & 2 & 1 & 3 \\
\hline Total & 10 & 10 & 20 \\
\hline
\end{tabular}

\section{Discussion}

Bladder carcinoma is more prevalent in developed countries. The most common type of bladder carcinoma is transitional cell carcinoma with its differentiated type. TURBT is the most common procedure performed in these patients with a good result. The problem associated with this technique is the fear of obturator nerve reflex thereby leading to bladder perforation. ${ }^{8,9}$

Among the various known risk factors, cigarette smoking is the most important and responsible for $48 \%$ cases of bladder cancer in men and $32 \%$ in female in USA. ${ }^{10}$ Factors that may increase chance of developing bladder cancer include chronic bladder inflammation or infection, exposure to arsenic, chemotherapeutic drugs, radiation treatment of the pelvis etc. The incidence and prevalence rates increase with age, peaking in the 8th decade of life with males being 3 to 4 times more likely to develop bladder cancer than females. Hematuria occurs commonly in bladder cancer and is present in most of the patients. Other signs and symptoms include frequent and painful urination and lower back pain.

TURBT is a procedure that is commonly done under spinal anaesthesia. It generally causes blockage of sensory pathway but the adductor jerks may occur due to the motor component. Magora F, Rozin R et al,1969 carried out fourteen obturator nerve blocks, in 8 patients, by blind anatomical approach, as controlled by imageintensifier fluoroscopy and electrical stimulation. ${ }^{11}$ In his study it was concluded that the anatomical approach which is controlled by the electrical stimulation of nerve is a much more accurate method as compared to the blind procedure. According to Augsurqer et al obturator block given by blind approach blocked about $85 \%$ of obturator nerve reflexes and the results were better if the block was given using nerve stimulation. ${ }^{12,13}$ According to Gasparich et al. and Kobayashi et al ONB using nerve stimulation techniques was $100 \%$ effective in only one case. ${ }^{14}$ Kobayashi et al userd the nerve method by injecting about $25 \mathrm{ml}$ of local anaesthetic with about 90 $\%$ success rate. ${ }^{14}$

ONB with the help of nerve stimulator is a much more accurate method of giving the anatomical block. However the major disadvantage is the excess of time utilized in this process as compared to blind procedure. In our study success rate of blind ONB was $80 \%$. Malik MA, Nawaz S, Khalid M, et al 2013, noted success rate of blind ONB to be about $90 \%$ while Augspurger et al had a success rate of about $85 \%$ which is comparable to our study. ${ }^{13-15}$

In our study, success rate of nerve stimulator guided obturator nerve block was $90 \%$. Gasparich et al and Kobayashi et $\mathrm{al}^{16}$ reported that ONB with nerve stimulator has better success rate. ${ }^{16,17}$ This was also comparable to our study.

\section{Conclusion}


The effectiveness of blind technique of obturator nerve block (Group 1) in our study was $80 \%$ while that via nerve stimulator guided technique (Group 2) was $90 \%$. This study indicates that obturator nerve block with anatomical technique has comparable success and complication rate, however use of nerve stimulator before ONB has better outcome.

\section{References}

1. Cancer Incidence in Five Continents. Vol .VIII (155). Lyon, France, IARC Publications, 2002. Edited by D.M. Parkin, S.L. Whelan, J. Ferlay, L. Teppo and D.B. Thomas

2. "SEER Stat Fact Sheets: Bladder Cancer". NCI 2014.

3. CAMPBELL WALSH UROLOGY 10TH Edition (Jemal et al, 2008;Parkin, 2008).

4. "European Association of Urology (EAU) - GuidelinesOnline Guidelines". Uroweb.org.

5. Wheeler ME, Weinstein SL: Adductor tenotomyobturator neurotomy. J Pediatr Orthop 4:48-51, 1984.

6. Pelissier J 1993: Chemical neurolysis using alcohol in the treatment of spasticity in the hemiplegic. Cah Anesthesiol 41;139-43.

7. Benzel EC, Barolat-Romana G, Larson SJ: Femoral obturator and sciatic neurectomy with iliacus and psoas muscle section for spasticity following spinal cord injury. Spine 13:905-8, 1988.

8. Junne-Yin Kuo; Prevention of Obturator Jerk during Transurethral Resection of Bladder Tumor, JTUA 19:2731, 2008.

9. Mydlo JH, Weinstein R, Shah S, Solliday M, Macchia $\mathrm{RJ}$; Long-term consequences from bladder perforation and/or violation in the presence of transitional cell carcinoma: results of a small series and a review of the literature. J Urol 1999;161:1128-32.

10. Kirkali Z, Chan T, Manoharan M, Algaba F, Busch C, Cheng L, Kiemeney L, Kriegmair M, Montironi R, Murphy WM, Sesterhenn IA, Tachibana M, Weider J. Bladder cancer: epidemiology, staging and grading, and diagnosis. Urology 2005;66 (Suppl 6A):434

11. Magora F,Rozin R et al,1969, "obturator nerve block: an evaluation of technique”, br. J. Anaesth. (1969), 41, 695.

12. "Bladder Cancer Stages, Prognosis, Diagnosis, and Treatment". WEB-MD Website.

13. Augspurger RR, Donohue RE. ,"Prevention of obturator nerve stimulation during transurethral surgery", J Urol. $1980 \mathrm{Feb} ; 123(2): 1702$

14. Junne-Yin Kuo; Prevention of Obturator Jerk during Transurethral Resection of Bladder Tumor, JTUA 19:2731, 2008.

15. Malik M A, Nawaz S, Khalid M, Et Al. "Obturator Nerve Block (Onb) In Transurethral Resection of Bladder Tumor (Turbt)" Jumdc Vol. 4, Issue 2, July-December 2013.

16. Kobayashi M et al 1991, "A report on 107 cases of obturator nerve block”, Masui. 1991 Jul;40(7):1138-43

17. Gasparich JP, Mason JT, Berger RE; Use of nerve stimulator for simple and accurate obturator nerve block before transurethral resection. J Urol 1984;132:291-3. 